\title{
EDITORIAL
}

\section{Rethinking Stable Ischemic Heart Disease: Time for a Copernican Revision}

Cardiovascular diseases in general and chronic myocardial ischemia in particular represent major public health concerns that continue to have a poor prognosis. Stunning advances of past decades in identification and quantification of obstructive coronary artery disease (CAD) stenosis represent the success of the traditional belief that this condition underlies essentially all stable ischemic heart disease (IHD) syndromes. The real question is whether we can improve a patient's quality of life with the minimum risk. It follows that, once a patient at high prognostic risk has been identified (usually by exercise testing and subsequent angiography); we have time to optimize the management of those at lower risk. Thus time should be taken to address lifestyle issues and utilize drug therapy based on the evidences. Over the last 30 years considerable progress has been made in the treatment of ischemic heart disease (IHD). However, population studies confirm that the problem is far from being solved, and IHD remains the leading cause of morbidity and mortality. Compared with the past, today's patients tend to be older, to have undergone revascularization procedures, and more often to have comorbidities, including heart failure and diabetes. However, there are now many "loose ends" that challenge this preoccupation equating obstructive stenosis with IHD, suggesting that it may be time to rethink this scientific paradigm.

\section{Coronary atherosclerotic obstructions and ischemic} heart disease: an elusive link.

The clinical approach to ischemic heart disease (IHD) is currently based on the dogmatic theory that coronary insufficiency secondary to atherosclerotic obstruction of coronary arteries is the unique cause of myocardial ischemia. Therefore, ruling out coronary artery disease at coronary angiography (ICA) is regarded as a key point in the stepwise evaluation of patients with symptoms and/or signs of myocardial ischemia, and a large amount of resources has been invested in developing noninvasive tests to better predict the presence of significant coronary stenosis at ICA. Several recent reports strongly challenge this approach because they have found a very poor association between coronary stenosis and myocardial ischemia.
Cheng et al have examined coronary anatomy with computed tomographic angiography (CTA) and reported that less than $50 \%$ of male patients older than 70 years and complaining of typical angina have significant stenosis. Moreover, the prevalence of coronary stenosis in patients with typical angina is similar to that observed in asymptomatic patients, and in both sexes it appears to only depend on the patient's age. Naya et al investigated the quantitative relationship between coronary atherosclerotic plaques assessed with CTA, and myocardial perfusion measured with positron emission tomography. In their report, they mentioned that myocardial perfusion can be normal downstream of a tight stenosis and abnormal downstream of a fully patent vessel. Moreover, patients with $0 \%$ stenosis diameter or a summed stenosis score of 0 by CTA may have abnormal myocardial flow reserve (MFR), and patients with $70 \%$ stenosis may present with normal MFR.

With an invasive approach, Park et al confirmed that fractional flow reserve (FFR), measured by pressure wire, may be in the normal range ( 0.80$)$ even in vessels with a markedly reduced minimal luminal area (MLA) on intravascular ultrasound. Thus, $63 \%$ of lesions with an MLA $<2.4 \mathrm{~mm}^{2}$ have an FFR (0.80) and even patients with the minimal diameter measurable $(<1 \mathrm{~mm})$ may have a normal FFR. The consistent observation in these papers is that myocardial perfusion cannot be predicted by coronary anatomy, despite the accuracy of the measuring tools. These studies also conclusively demonstrate that the majority of patients with angina pectoris and/or myocardial ischemia do not have significant coronary stenosis.

Furthermore, the persistence of angina and inducible ischemia after percutaneous or surgical coronary recanalization, underscore the fact that removing a stenosis in stable IHD patients does not consistently remove the pathologic mechanism that causes myocardial ischemia. In summary, based on this evidence, the theory of a selective and exclusive link between coronary atherosclerotic obstructions and IHD is no longer defensible. The clinical approach to IHD should now be based on the awareness that IHD is a 
multi-factorial disease and that myocardial ischemia can also be precipitated by a number of mechanisms, including coronary vasospasm, thrombosis, inflammation, coronary microvascular dysfunction, endothelial dysfunction, etc (Fig. 1).

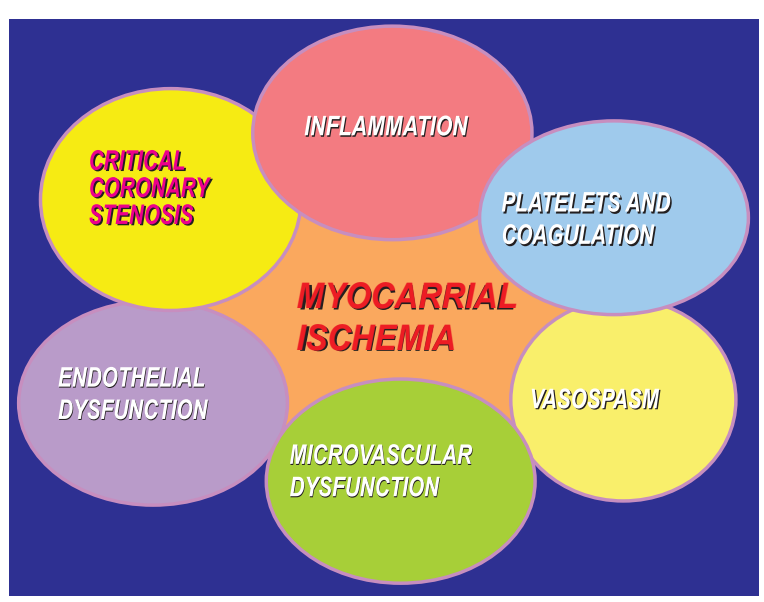

Fig.-1: The proposed shift in paradigm ("the solar system of ischemic heart disease") puts myocardiaal ischemia at the center.

This shifts the focus away from obstructive coronary atherosclerosis and puts it on the coronary microvasculature and the myocardial cell, where ischemia actually takes place. If the cardiomyocytes are placed at the center of the model, a number of potential pathological inputs come into play and strategies that protect the cardiomyocytes from ischemic damage can be developed, regardless of the causative mechanism.

In the absence of obstructive coronary artery disease (CAD), management of patients with angina represents an unsolved challenge. Such patients are often labeled as either patients with "atypical angina" or patients with "false positive results" at noninvasive evaluation. However, long-term follow-up studies suggest that patients with angina and ischemia, who do not display obstructive CAD, have increased coronary event rates and adverse quality of life as compared with those with no evidence of ischemia, underscoring the relevance of proper identification and treatment.

In this current situation our first step is to recognize the growing evidence against the unitary theory of flowlimiting stenosis as the prerequisite for stable ischemic heart disease and to accept the need to readjust the prevailing wisdom of myocardial ischemia. The model proposed herein shifts the focus away from obstructive epicardial coronary atherosclerosis and centers it on the microvasculature and myocardial cell where the ischemia is taking place and in doing so, call for a revision using "the Copernican" revolution metaphor. Given the inconsistencies associated with the obstructive "plaquecentric" approach, a shift in the paradigm that takes into consideration the multifactorial aspect of IHD seems warranted. Our attention should be focused on the microvascular with resultant myocardial ischemia and on the myocardial cell. If we put the myocardial cell at the center of the model, all the potential pathological inputs that might drive progression to unstable angina, acute myocardial infarction, and sudden cardiac death can be considered, starting with obstructive atherosclerosis but also including inflammation, endothelial dysfunction, microvascular dysfunction, platelet dysfunction, thrombosis, and vasomotor dysfunction. In daily practice, such a shift in focus could imply either identifying mechanisms responsible for ischemia and applying a specific treatment in each patient or developing strategies that can protect the cardiomyocytes from ischemic damage, regardless of the causative mechanism. If the myocardial cell is placed at the center of the model, all the potential pathological inputs can be considered, and strategies that protect the cardiomyocytes from ischemic damage, regardless of the causative mechanism, can be developed.

(J Banagladesh Coll Phys Surg 2013; 31: 179-180)

\section{Prof. Mohammad Nazrul Islam \\ MBBS, FCPS, FRCP, FACC, FESC \\ UGC Professor, Department of Cardiology and \\ Course Director, Faculty of Medicine; BSM Medical University}

\section{References:}

1. Gould KL, Parsel L et al. Assessment of coronary stenosis buy myocardial perfusion imaging. Am J Cardiol. 1974; 33(1): 87-94.

2. Patel MR et al. Rivaroxaban versus warfarin in nonvalvular atrial fibrillation 2011; 365(10): 883-91.

3. Cheng VY et al. CONFIRM Study. Circulation. 2011; 124(22): 2423-32.

4. Naya M. Foster CR. Coronary vascular dysfunction and prognosis in patients with chronic kidney disease. 2011; 58: 1989-1997.

5. Park SJ et al. Paradigm shift to functional angioplasty. 2011; 124: 951-957.

6. Boden WE et al. Should we review our approach to optimal medical therapy ? N Engl J Med. 2007; 35: 1503-1516.

7. Marzilli M. Optimal medical therapy with or without PCP for stable coronary disease. 2012; 60(11): 957-959. 